Tatiana Bachniak ${ }^{1}$

\title{
Die Regel der Übersetzung nach Hieronymus in der Theorie und Praxis
}

\section{Einführung}

Hieronymus von Stridon (347-30.09.419/420)2, den die katholische Kirche zum Rang des großen lateinischen Kirchenvaters und des Patrons der Theologen, Gelehrten, Schüler, Studenten, Lehrer, Übersetzer und Korrektoren erhoben hatte ${ }^{3}$, ist dank seines enormen und mannigfaltigen literarischen Erbes auf vielen, nicht nur theologischen Arbeitsgebieten als Autorität zu betrachten. Vor allem aber ist er als „Mann der Bibel“ in die Geschichte eingegangen. Sein Werk, die Vulgata, galt viele Jahrhunderte als offizieller Bibeltext der Katholischen Kirche ${ }^{4}$, als Grundlage für jegliche katechetische, kerygmatische, missionarische und nicht zuletzt theologische Tätigkeit.

$\mathrm{Zu}$ Beginn des großen Vorhabens stand der Auftrag einer Revision der vielen verbreiteten lateinischen Evangelien-Versionen. Damit seine Arbeit

1 Tatiana Bachniak, doktorantka w Katedrze Historii Kościoła i Patrologii Wydziału Teologicznego Uniwersytetu Opolskiego, e-mail: tatiana.bachniak@gmail. com, ORCID: 0000-0003-2556-1034.

2 Biographische Angaben in Kurzform s. M. Durst, Art. Hieronymus I, LThK 5, 91, Freiburg in Breisgau 2006, Sonderausgabe oder A. Fürst, Art. Hieronymus I, LACL, Freiburg in Breisgau 2002, 323-324. Detaillierte Darstellung aller Daten und Fakten aus dem Leben Hieronymus, sowie ausführliche Literaturangaben präsentiert A. Fürst, Hieronymus. Askese und Wissenschaft in der Spätantike, Freiburg in Breisgau 2003, 145-149.

3 S. Art, Hieronymus, in: Lexikon der Heiligen, Genehmigte Lizenzausgabe, Augsburg 2006, 225.

4 S. Art, Bibel VIII, LThK 2, 384. 
unvoreingenommen und unbeeinflusst werden konnte, entschloss sich Hieronymus auf die griechischen Quelltexte zurückzugreifen. Des weiteren folgte die Übersetzung der alttestamentlichen Schriften nach ihren hebräischen Vorlagen und nicht wie bisher nach der Septuaginta. Bei solchem Unterfangen stand Hieronymus vor der Entscheidung, ob die Übertragung wortwörtlich oder sinngemäß erfolgen sollte. Inwieweit durfte er sich von den gängigen und vertrauten Versionen entfernen ohne zu viel Verwirrung bei den Lesern hervorzurufen.

In diesem Artikel soll anhand der zahlreichen, sich oft zu widersprechen scheinenden Äußerungen des Kirchenvaters eine allgemeine ${ }^{5}$ Regel dargestellt werden, die einerseits als maßgebend für seine translatorische Vorgehensweise gelten, andererseits den jeweiligen Umständen angepasst werden kann.

\section{Vir trilinguis ${ }^{6}$}

Der Lauf der klassischen Ausbildung, die Hieronymus in Rom bei dem berühmten Grammatiklehrer Aelius Donatus genossen hatte ${ }^{7}$, der in ihm die Liebe zu den großen antiken Autoren wie Cicero, Vergil, Terenz, Horaz oder Historiker Sallust samt ihrer Werken entflammt hatte, nahmen großen Einfluss auf seinen eigenen Schreibstil, der bis heute als hervorragend und beispielhaft gilt ${ }^{8}$. Hieronymus war im Besitz zahlreichen Abschriften der klassischen Literatur, die er teils eigenhändig angefertigt, teils für großes Geld erworben hatte9. Er selbst bezeugte in einem Brief an Eustochium, dass er sich von seiner mit Fleiß und Mühe angelegten Bibliothek nicht trennen konnte und sie überall mit auf die Reisen nahm ${ }^{10}$.

5 Eine interessante Darstellung der Quellen und der ganzen Arbeitsmethode bei der Übersetzung des Buches Deuteronomium präsentiert S. Weigert in seiner Dissertation Hebraica Veritas. Übersetzungsprinzipien und Quellen der Deuteronomiumübersetzung des Hieronymus, Stuttgart 2016. Der Autor stellt dabei den aktuellen Forschungsstand zu diesem Thema dar.

6 S.S. Rebenich, The "Vir Trilinguis" and the "Hebraica Veritas", VigChr 47 (1993) 50-77.

7 S.H. Graf Reventlow, Epochen der Bibelauslegung, Bd. 2: Von der Spätantike bis zum ausgehenden Mittelalter, München 1994, 39; Fürst, Hieronymus, S. 60-65.

8 Reventlow, Epochen, S. 39.

9 Fürst, Hieronymus, S. 65-66.

10 S. Ep. 22, 30. 
Gereist ist Hieronymus viel. Seine längere Aufenthalte in den griechischsprächigen Gegenden wie Antiochia (zw.373/74-379/80) ${ }^{11}$, wo er Griechisch gelernt und bei Appolinarius von Laodicea die Arkana der antiochenischen Bibelexegese kennen gelernt hatte ${ }^{12}$; des weiteren Konstantinopel (379/80-382) ${ }^{13}$, dem Bischofsitz des Gregor von Nazianz, wo er fleißig griechische Exegese studierte, um sich daraufhin als Übersetzer zu versuchen ${ }^{14}$; und schließlich Alexandria (386) ${ }^{15}$, die Stadt des berühmten Bibelexegeten Didymos des Blinden, dessen Hauptwerk „Über den Heiligen Geist“ dankt der lateinischen Übersetzung des Hieronymus bis heute erhalten blieb ${ }^{16}$, waren die besten Voraussetzungen für das erreichte hohe Niveau seiner klassischen Sprachkenntnisse. Darüber war sich Hieronymus im bewussten, wenn er um das Jahr 392/393 schrieb: ,[...] Und was das Lateinische angeht, so habe ich mein Leben nahezu von der Wiege an unter Grammatikern, Rhetoren und Philosophen zugebracht $[\ldots]^{6 / 17}$.

Dazu kam das Verlangen nach neuen Herausforderungen, was sich in seinem Interesse für das Hebräische, Aramäische und Syrische zeigte ${ }^{18}$. Während des Aufenthalts in der Wüste Chalkis (375-377) ${ }^{19}$, auf einem Landgut seines Freundes Evagrius, der ihm eigene Bibliothek, Schreiber und Kopisten zur Verfügung stellte, konnte Hieronymus seine biblischen Studien fortführen. Dort knüpfte er Kontakte zu einem konvertierten Juden, bei dem er die ersten Schritte im Hebräischen stellte. Ursprünglich betrachtete Hieronymus das Erwerben der hebräischen Sprache als eine asketische Übung, die ihn von den Gedanken an das Fleischliche und Leidenschaftliche fern hielt. Rückblickend schreibt er an den Mönch Rusticus (um 409) folgende Worte:

Als mich in meinen jungen Jahren die Einsamkeit der Wüste schützend umgab, da konnte ich der Anreiz zum Laster und die in der Natur begründete Glut der Sinn nicht mehr ertragen. Obwohl ich sie durch häufiges Fasten

11 Fürst, Hieronymus, S. 145.

12 Reventlow, Epochen, S. 40.

13 Fürst, Hieronymus, S. 146.

14 Fürst, Hieronymus, S. 146; L. Schade, Die Inspirationslehre des Heiligen Hieronymus. Eine biblisch-geschichtliche Studie, Freiburg im Breisgau 1910, 104.

15 Fürst, Hieronymus, S. 146; Schade, Die Inspirationslehre, S. 105.

16 Hieronymus selbst erwähnt das Werk Didymos' in einem Brief an den Papst Damasus, dem er seine Übersetzung widmet (Ep. 36, 1). S. auch B. Kramer, Art. Didymos der Blinde, LThK 3, 213.

17 Vulg. Ijob Prol. Übersetzung Fürst, Hieronymus, S. 271.

18 S.D. Brown, Vir trilinguis, A Study in the Biblical Exegesis of Saint Jerome, Kampen 1992, 82-85.

19 Fürst, Hieronymus, S. 48. 
gebrochen hatte, brodelte es in meiner Phantasie noch immer von bösen Vorstellungen. Um sie zu überwinden, ging ich zu einem Bruder, der aus dem Judentum Christ geworden war, in die Lehre. [...] Und ich lernte jetzt das Alphabet und studierte die hebräischen Vokabeln mit ihren Zisch- und Kehllauten. Was für eine Anstrengung dies kostete, welche Schwierigkeiten zu überwinden waren, wie oft ich verzweifelte, wie oft ich die Sache drangab und voller Lernbegierde wieder aufnahm, das weiß nur ich, der ich es durchgemacht habe, und jene, die mit mir zusammenlebten. Und heute danke ich Gott, dass ich aus dieser bitteren Buchstabensaat so herrliche Früchte einheimsen kann $^{20}$.

Später, nach der Niederlassung in Bethlehem (386) setzt Hieronymus den Hebräischunterricht fort. In einem Brief an seine römischen Freunde Pammachius und Oceanus ${ }^{21}$ erwähnt er einen Juden Namens Baraninas, der ihn nachts besucht um ihn $\mathrm{zu}$ unterrichten ${ }^{22}$. Mehrfach bringt Hieronymus selbstbewusst die Überzeugung über seine zufrieden stellende Hebräischkenntnisse zum Ausdruck. Im Prolog zu der Übersetzung der Königsbücher, die um das Jahr 390 entstanden ist, wendet er sich voll Ironie an den Leser mit der Aufforderung, einen Hebräer seines Vertrauens zu konsultieren, wenn er vermutet, dass Hieronymus etwas an der hebraica veritas geändert hätte ${ }^{23}$.

Im gleichen Geist äußert sich unser Autor im Prolog zum Pentateuch, den er um das Jahr 400 verfasst hatte, als er seinem vermeintlichen Neider und Kritiker den Vorschlag erteilt, sich bei einem Hebräer oder den Rabbinern in der Stadt eine Meinung zu holen, um zu verifizieren, ob die Übersetzung des Hieronymus korrekt, oder ob ihm ein Fehler unterlaufen $\operatorname{sei}^{24}$.

\section{3. Übersetzung aus der Quelle}

Der Eifer, mit dem Sophronius Eusebius seine Hebräischkenntnisse verteidigt und sich ihrer rühmt ${ }^{25}$, ist nachvollziehbar, wenn man bedenkt, welche Hochachtung er dem Hebräischen entgegenbringt. Hieronymus hält

\footnotetext{
20 Ep. 125, 12. Übersetzung Schade, BKV.

21 Ep. 84, 3.

22 S. auch Reventlow, Epochen, S. 44.

23 Fürst, Hieronymus, S. 268.

24 Fürst, Hieronymus, S. 277.

25 Brown, Vir trilinguis, S. 71-82.
} 
Hebräisch, gemäß der Tradition, für den Ursprung aller Sprachen ${ }^{26}$. Doch selbst diese Auffassung hielt ihn nicht davon ab, die Sprache des Alten Testaments gelegentlich als barbarisch zu bezeichnen ${ }^{27}$. Als Mann der Bibel, für den das größte Glück darin bestand, im Gesetz des Herren Tage und Nächte zu forschen (Ps 1,2), seine Geheimnisse zu ergründen, Gottes Pläne in der Heiligen Schrift zu erkennen und somit zur Weisheit des Geistes zu gelangen ${ }^{28}$, wurde Hieronymus allmählich zu einem gefragten Spezialisten auf dem Gebiet der biblischen Exegese. Die zahlreiche Korrespondenz zwischen dem Papst Damasus (366-384) und dem vir trilinguis ${ }^{29}$ vermittelt ein Bild des Wissenschaftlers, der dem Papst die schwierigen und unklaren Bibelstellen erklärt ${ }^{30}$. Bei dieser Gelegenheit weist Sophronius Eusebius sehr oft auf die Tatsache hin, dass die verschiedenen griechischen und lateinischen Übersetzungen der Bibel von dem hebräischen Original abweichen, was die Ursache der Unklarheiten sei ${ }^{31}$. In Zweifelfällen sollte man immer zu der Quelle greifen, so Hieronymus ${ }^{32}$, was im Fall des Neuen der griechische, mit Ausnahme des Ur-Matthäusevangeliums (das sog. Hebräerevangelium, das Hieronymus aus dem Aramäischen ins Griechische und Lateinische übertragen hatte ${ }^{33}$ ), im Fall des Alten Testaments der hebräische Urtext ist. Von dieser Korrespondenz ermutigt und durch Damasus selbst angespornt ${ }^{34}$ stellte sich Hieronymus einer großen Herausforderung. Was im Jahr 382 mit einer Revision der verschiedenen voneinander abweichenden Evangelien-Versionen, die sich im Laufe der Jahrhunderte im kirchlichen Gebrauch vermehrt hatten, angefangen hatte, wuchs zu einer Lebensaufgabe der komplett neuen Bibelübersetzung anhand der Quelltexte heran. Die Notwendigkeit der Neuübersetzung ergab sich aus der Tatsache, dass es in den Evangelien viele alttestamentliche, von Jesus zitierte oder von den Aposteln niedergeschriebene Schriftworte gab, die in den vorhandenen Septuaginta-Texten nicht zu finden waren. Zahlreiche Beispiele solcher Zitate führt Hieronymus unter anderem im Prolog zu den Chronikbüchern (395/396), im Brief 57, 7-9 aus dem Jahr 396 und im Prolog zum Pentateuch (um 400) auf. Ein interessantes Werk,

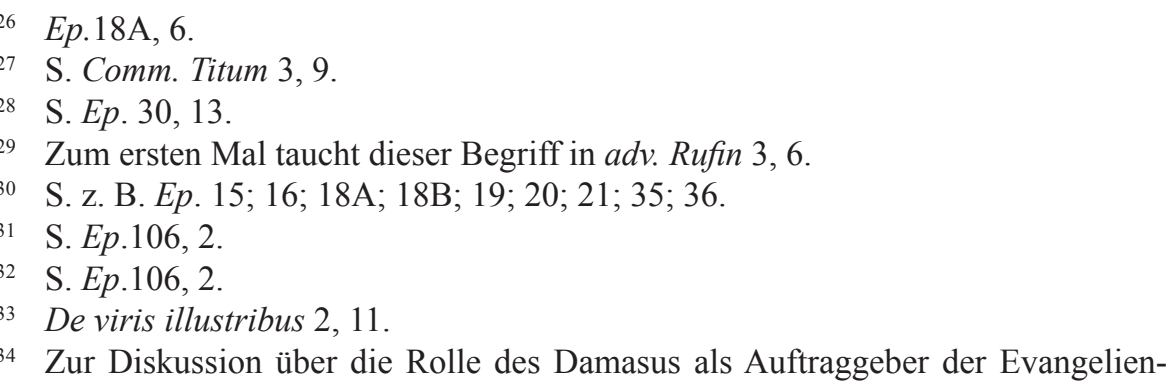


das sich mit der Analyse des Psalters beschäftigt und sämtliche Differenzen zwischen den griechischen und lateinischen Übersetzungen mit den hebräischen Quellen vergleicht, ist ein zwischen 403-410 entstandener Brief an Sunnia und Fretela ${ }^{35}$. Diese Schrift schildert die Arbeitsweise des Hieronymus anhand der biblischen Texte. Der Kirchenvater scheut keine Mühe, um die einzelnen Worte, ihre Etymologie und die Bedeutung mithilfe anderer Bibelstellen den interessierten Lesern zu erklären und nahe zu bringen.

Um den Originaltext, den er seiner translatorischen Arbeit zu Grunde legen wollte, eruieren zu können, strebte Hieronymus danach, zu den Quellen, ad fontes, zu gelangen, um sich nicht mehr mit den unterschiedlichsten Übersetzungen rivuli - die Bächlein, zufrieden geben zu müs$\operatorname{sen}^{36}$.

Während im Fall der Evangelien diese Quelle in griechischer Sprache verfasst wurde ${ }^{37}$, gilt für das Alte Testamen ab Mitte der 390-er Jahre der Begriff hebraica veritas, der sich auf den auf hebräisch niedergeschriebenen Bibeltext bezieht ${ }^{38}$.

Der Begriff selbst ist in zweifacher Hinsicht zu interpretieren: einerseits philologisch-textkritisch, wo er soviel wie „Urtext, Vorlage, unverfälschtes Original, ${ }^{39}$ bedeutet, andererseits sprachphilosophisch im Bezug auf die göttliche Inspiration des hebräischen Textes, der das Wort Gottes und seine Verheißung offenbart ${ }^{40}$.

So lesen wir in einem Brief an Damasus, in dem die Bedeutung des Wortes „Hosianna“ erklärt wird, dass man sich in diesem Fall der Quelle zuwenden soll, denn nur dort wird man die Wahrheit finden ${ }^{41}$.

Das hohe Ansehen, das Hieronymus bei seiner Arbeit dem hebräischen Bibeltext beigemessen hatte, machte den Kirchenvater zur Zielscheibe für diejenigen, die ihm vorwarfen, er wolle dem hoch angesehenen Text der Septuaginta, den Rang und den inspirierten Charakter abstreiten. Gegenüber seinen Kritikern - den realen und den potenziellen - wies Hieronymus mehrfach entschlossen diese Vorwürfe zurück. Im Vorwort zu seinen Untersuchungen zur hebräischen Sprache im Buch Genesis schreibt er um das Jahr 391/392:

35 Ep. 106.

36 S. Ep. 106, 2. Zum Ursprung dieses Bildes s. Weigert, Hebraica veritas, Anm. 83,31 .
37 S. Ep. 71, 5.
38 Weigert, Hebraica veritas, S. 32-40.
39 Weigert, Hebraica veritas, S. 32.
40 Weigert, Hebraica veritas, S. 32.
41 S. Ep. 20, 2. 
Unser Ziel wird es daher sein, einerseits die Fehler derer aufzudecken, die über die hebräischen Bücher die unterschiedlichsten Mutmaßungen vorbringen, andererseits den wild wuchernden Text in den lateinischen und griechischen Handschriften in eine authentische Form zu bringen. [...] Wir zerren damit allerdings nicht, wie unsere Neider geifern, die Fehler der Siebzig Übersetzer in den Anklagestand noch betrachten wir unsere Arbeit als Kritik an ihnen $[\ldots]^{42}$.

Im Prolog zu der Übersetzung des Buches Hiob aus dem Jahr 392/93 lesen wir:

Bei jedem einzelnen Buch der göttlichen Schrift sehe ich mich gezwungen, den Schmähungen meiner Gegner entgegenzutreten, die mir vorwerfen, mit meiner Übersetzung würde ich die Siebzig Übersetzer in Frage stellen $[\ldots]^{43}$. Hört also meine bellenden Kritiker! Mit der Arbeit an diesem Buch verfolge ich nicht die Absicht, die alte Übersetzung in Frage zu stellen. Vielmehr sollten diejenigen Stellen, die in jener unklar sind, die fehlen oder die jedenfalls aufgrund von Abschreibfehlern verderbt sind, durch meine Übersetzung klarer werden ${ }^{44}$.

In der Tat bemüht sich Hieronymus die im Laufe der Jahrhunderte mehrfach übersetzen und abgeschriebenen Bibeltexte näher an ihre Originalform zu bringen:

Das ist sicher eine gefährliche Arbeit, ausgesetzt dem Gekläff der Kritiker, die mir vorwerfen zur Verhöhnung der Siebzig Übersetzer würde ich Neues an die Stelle von Altem setzen und auf die se Weise Können prüfen wie Wein, obwohl ich schon überaus oft beteuert habe, dass ich mich lediglich nach Kräften bemühe, in das Heiligtum Gottes zu bringen, was ich kann (vgl. Ex 25,2-7; 35,5-9), und dass die Stärken der einen nicht durch die Unfähigkeit von anderen getrübt werden. [...] „Höre also, Neider! Kritiker, sperr die Ohren auf! Ich werfe und kritisiere die Siebzig nicht!“445

Aus den Aussagen ist deutlich zu erkennen, dass das große Vorhaben des Hieronymus nicht darauf abzielt, die LXX, der er aufgrund der Tatsache, dass sie von Jesus und von den Aposteln benutzt wurde, gewisse Hochschätzung entgegen brachte ${ }^{46}$, ihrer Anerkennung zu berauben.

\footnotetext{
42 Hebr. quest. in Gen. praef. (CCL 72,2). Übersetzung Fürst, Hieronymus, S. 264.

43 Vulg. Iob Prol. (Weber/Gryson). Übersetzung Fürst, Hieronymus, S. 269.

44 Fürst, Hieronymus, S. 271.

45 Vulg. Pent. Prol. (Weber/Gryson). Übersetzung Fürst, Hieronymus, 275, 277.

46 S. Ep. 57, 11.
} 
Vielmehr lag es ihm daran, einen zuverlässigen Bibeltext zu ermitteln, der der Auseinandersetzung mit dem Judentum Stand halten würde, sodass die Kirchenmänner in Gesprächen mit den Juden nicht mehr die Gefahr laufen, aufgrund der Differenzen zwischen den ihnen zugänglichen SeptuagintaÜbersetzungen und den Texten der jüdischen Gelehrten von den Letzteren ausgelacht $\mathrm{zu}$ werden ${ }^{47}$, des Weiteren auch als Grundlage für jegliche exegetische Arbeiten, deren Voraussetzung die Kenntnis der hebräischen Sprache ist ${ }^{48}$, ohne Bedenken verwendet werden könnte.

Die persönliche Stellung des Hieronymus gegenüber der LXX wandelte sich jedoch im Laufe seiner translatorischen Beschäftigung ${ }^{49}$. Während er anfangs die Meinung vertrat, die Abweichungen zwischen dem hebräischen Originaltext und der Übersetzung der Siebzig ergaben sich aus der Tatsache, dass der Auftraggeber, Ptolemäus II, nicht die ganze Wahrheit der göttlichen Offenbarung erfahren durfte ${ }^{50}$, so glaubte er mit der Zeit an die willentliche Verschleierung der Übersetzung, die ausschließlich aus der Perspektive des Erlösungsgeheimnisses Jesu Christi vollständig und korrekt entschleiert und, was daraus folgt, auch übersetzt werden könne:

Was nun? Verwerfen wir die Alten? Keineswegs! Wir bemühen uns vielmehr, nach der Arbeit der früheren Übersetzer im Hause des Herren zu leisten, was wir können. Jene fertigten ihre Übersetzung vor der Ankunft Christi an, und was sie (noch) nicht verstanden haben, formulierten sie mit ungenauen Ausdrücken. Wir hingegen bringen nach seinem Leiden und seiner Auferstehung weniger Verheißenes als vielmehr geschehenes aufs Papier. Was man selbst gesehen hat, das erzählt man anders als das, was man nur gehört hat, und was wir besser verstehen, das können wir auch besser formulieren ${ }^{51}$.

Für die seelsorgerische Arbeit, wie z.B. der Psalmengesang in den kirchlichen Gemeinden ${ }^{52}$, ließ Hieronymus nach wie vor die Verwendung des Septuagintatextes aus Rücksicht auf die weitläufige Tradition und den daraus resultierenden Vertrauensfaktor der Gläubigen $\mathrm{zu}^{53}$.

47 Über solche Vorfälle berichtet Hieronymus z. B. in Praef. PS (IH), und in Prol. Hier. (IH). S. auch Reventlow, Epochen, S. 46.

48 Diese Meinung äußert Hieronymus in Ep. 106, 46.

49 Ausführliche Darstellung dieses Problems liefert Schade, Die Inspirationslehre, S. 141-157.

50 Diese Überzeugung finden wir z. B. in Prol. Ijob; Prol. Pent.

51 Prol. Pent. Übersetzung Fürst, Hieronymus, S. 276-277.

52 S. Ep. 106, 46.

53 Eine interessante Darstellung dieser Problematik liefert E. Schulz-Flügel, Hieronymus. Feind und Überwinder der Septuaginta? Untersuchungen anhand der 
Im Kommentar zum Buch Kohelets aus dem Jahr 388/389, das als erstes vollkommen anhand hebräischen Grundlagen verfasstes Werk des Hieronymus gilt ${ }^{54}$, schreibt der Autor verantwortungsvoll im Vorwort:

Ich weise nur kurz darauf hin, dass ich keiner Vorlage (sc. existierender Übersetzung) gefolgt bin, sondern mich bei meiner Übertragung aus dem Hebräischen mehr an die gewöhnte Fassung der LXX- Übersetzer gehalten habe, zumindest dort, wo sie nicht allzu sehr vom Hebräischen abweicht. [...] So wollte ich weder den Eifer des Lesers durch allzu viel Neuheit verschrecken noch andererseits gegen mein Gewissen die Quelle der Wahrheit verlassen und den Bächlein der Meinungen folgen ${ }^{55}$.

Später (zwischen 406-410) in einem Brief an Sunnia und Fretela, wiederholt Sophronius Eusebius diese Einsicht beinahe wörtlich: „Wo immer der Sinn derselbe ist, wollten wir nicht die vertrauten Worte der alten Übersetzer ändern, um nicht durch übermäßige Neuerungen den Eifer des Lesers abzuschrecken" ${ }^{\text {"56 }}$.

Hieronymus war sich durchaus im Klaren darüber, welche Früchte seine Arbeit hervorbringen könne, und mit Sicherheit dachte er nicht an Ruhm und Ehre, sondern vielmehr an Kritik und Streit.

Zur Bestätigung dieser Vermutung sei ein Ereignis angeführt, das aus der Korrespondenz zwischen Hieronymus und Augustinus von Hippo zu entnehmen ist ${ }^{57}$. Es beschreibt die Verwirrung, die die neue Übersetzung des Propheten Jona aus der Feder des Hieronymus bei den Gläubigen, die an die Version der LXX gewöhnt waren, ausgelöst hatte.

Generell muss gesagt werden, dass die Gesinnung Augustinus`gegenüber der Leistung Hieronymus`als Übersetzer ambivalenter Natur war. Während er einerseits Hieronymus für seine Übersetzung der Evangelien

Arbeiten an den Psalmen, in: Septuaginta-Psalter und seine Tochterübersetzungen, red. A. Aejmelaeus - U. Quast, MSU 24, Göttingen 2000, 33-50; Hieronymus-Gottes Wort. Septuaginta oder hebraica Veritas, in: Die Septuaginta - Text, Wirkung, Rezeption, red. W. Kraus - S. Kreuzer, WUNT 325, Tübingen 2004, 746-758.

54 S.E. Birnbaum, Der KoheletKommentar des Hieronymus, Einleitung, revidierter Text, Übersetzung und Kommentierung, CSEL Extra Seriem, Berlin - Boston 2014, 2.

55 Übersetzung nach Birnbaum, Der Kohelet-Kommentar, S. 49.

56 Ep. 106, 12. Übersetzung S. Risse, Hieronymus, Commentarius in Ionam, lateinisch-deutsch = Kommentar zu dem Propheten Jona, Turnhout 2003, 24.

57 Es handelt sich um Ep. 71 des Augustinus, die um 403/403 in Bethlehem eintraf und die Antwort des Hieronymus in Ep. 112. Dazu s. Augustinus - Hieronymus, Epistulae mutae, lateinisch-deutsch $=$ Briefwechsel I, Übersetzt und eingeleitet von A. Fürst, FC 41/1, Brepols Publishers, Turnhout 2002. 
lobte und ihn inständig darum bat, eine Lateinübersetzung der alten, griechischen Kirchenlehrer, die Exegese betrieben hatten, anzufertigen, damit auch die Afrikanischen Bischöfe und Gelehrten, die des Griechischen nicht mächtig waren, ihre Werke lesen und ihre Einsichten kennen lernen könnten $^{58}$, so stellte er andererseits den Sinn der Neuübersetzung aus dem Hebräischen in Frage. Insbesondere war die angebliche Unfehlbarkeit Hieronymus', was die Hebräischkenntnisse angeht, dem Bischof von Hippo Regius ein Dorn im Auge: ,[...] Handelt es sich nämlich um unklare Stellen, ist anzunehmen, dass an ihnen auch Du Dich irren kannst" ${ }^{\text {"59 }}$.

Mit Hilfe der Schilderung des Ereignisses, das sich in der Stadt Oea (heute Tripolis) zugetragen haben sollte, wollte Augustinus dem Hieronymus vor Augen stellen, welch schwerwiegende Folgen seine translatorische Arbeit haben könne. Augustinus beschreibt, in welch große Schwierigkeiten der Bischof der Gemeinde geraten sein soll, als er in der Lesung aus dem Propheten Jona die Version des Hieronymus und nicht die vertraute und seit Generationen gehörte, aus der LXX stammende, verwendet habe. Die Gläubigen sollten mit großer Empörung und sogar mit dem Vorwurf einer Fälschung reagiert haben, sodass sich der Bischof gezwungen sah, nachdem die Klärungs-Versuche und die Befragung der Juden keine erhoffte Lösung brachten, die Textstelle zu korrigieren. Im Anschluss erlaubte sich Augustinus eigene Meinung dazu zu äußern, die er allerdings bereits früher vertrat: ,[...] Von daher meinen auch wir, dass bisweilen auch Du Dich an manchen Stellen hast irren können"60.

Die Antwort des Hieronymus und seine Stellungnahme zu den Vorwürfen erfahren wir aus dem Brief 112, 22:

Du behauptest, ich hätte im Propheten Jona etwas falsch übersetzt, und weil die Leute wegen eines einzigen anders lautenden Wortes lärmend in Aufruhr gerieten, habe ein Bischof beinahe sein Amt eingebüßt. Was ich freilich falsch übersetzt haben soll, das sagst Du nicht und nimmst mir damit die Möglichkeit, mich zu verteidigen und Deine wie auch immer gearteten Einwände zu widerlegen. Womöglich aber geht es, wie schon vor etlichen Jahren, erneut um den Kürbis. [...] Im Kommentar zum Propheten Jona habe ich dazu schon ausführlicher Stellung genommen. So beschränke ich mich jetzt auf den Hinweis, dass die siebzig Übersetzer

58 Augustinus. Ep. 28, 2; 116, 34. Auch bezüglich der Exegese vertraten die beiden Kirchenväter unterschiedliche Auffassung. Dazu s. R. Hennings, Der Briefwechsel zwischen Augustinus und Hieronymus und ihr Streit um den Kanon des Alten Testaments und die Auslegung von Gal. 2,11-14, VCSup 21, Leiden 1994.

59 Augustinus. Ep. 28, 2. Übersetzung Fürst, S. 103.

60 Augustinus, Ep. 71,5. Übersetzung Fürst. S. 165. 


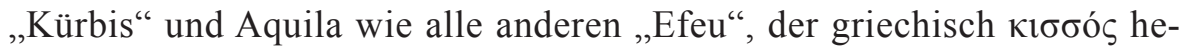
ißt, übersetzten. Im hebräischen Text steht an dieser Stelle ciceion, wofür die Syrer für gewöhnlich ciceia sagen. Es handelt sich um eine Pflanze mit breiten Blättern nach Art von Weinlaub, die nach dem Einpflanzen rasch zu einem kleinen Baum emporwächst ohne Stangen und Pfosten als Stützen, wie sie für Kürbis - und Efeusträucher vonnöten sind, gestützt allein vom eigenen Stamm. Hätte ich mich dafür entschieden, wortwörtlich zu übersetzen und ciceion zu transkribieren, würde das niemand verstehen. Mit „Kürbis“ würde ich ein Wort gebrauchen, das im Hebräischen nicht dasteht. In Übereinstimmung mit den übrigen Übersetzern habe ich also „Efeu“" in den Text gesetzt ${ }^{61}$.

In dem oben erwähnten Jonakommentar ${ }^{62}$ aus dem Jahr 391/392, den Hieronymus seinem alten Freund Chromatius ${ }^{63}$ widmete, polemisiert unser Autor mit einem gewissen Canterius, von dem er in Rom „des Sakrilegs" angeklagt worden sei $^{64}$. Der Grund dafür war die Übersetzung der Stelle Jona 4,6, wo die Schattenspendende Pflanze nicht wie bislang „Kürbis“, sondern „Efeu“ genannt wurde ${ }^{65}$. Auf seine typische ironisch-sarkastische Weise ${ }^{66}$ versucht Hieronymus den Anklagegrund seines Widersachers lächerlich zu machen, gleichzeitig verteidigt er mit Nachdruck seine Pflicht der Treue gegenüber dem hebräischen Original ${ }^{67}$.

Bei dieser Gelegenheit wird der Leser mit einem anderen Grundsatz der Übersetzungskunst konfrontiert. Das Anliegen, ob man die Bibel wortwörtlich oder sinngemäß übersetzen sollte, war für Sophronius Eusebius von entscheidender Bedeutung. Im Fall des Jonakommentars handelte es sich lediglich um eine Pflanzenart, die im Lateinischen kein Äquivalent besäß $\mathrm{e}^{68}$. Wie verhielt es sich mit der ganzen Heilsbotschaft, die die Bibel zu vermitteln hat?

${ }^{61}$ Ep. 112, 12. Übersetzung Fürst, S. 227-229. Ausführlicher dazu: A. Fürst, Kürbis oder Efeu?, BN 72 (1994) 12-19.

62 Es handelt sich um die Stelle In Ionam IV, 6.

63 S. Praef. Ad Chrom.; Zur Person des Chromatius s. Fürst, Hieronymus, S. 164-165.

64 S. In Ionam IV, 6.

65 S. In Ionam IV, 6.

66 Zum Stil der hieronymischen Polemik s. I. Opelt, Hieronymus 'Streitschriften, Heidelberg 1973, 155-187.

67 S. In Ionam 4, 6.

68 A. Fürst entlarvt diese Aussage als falsch, denn die Pflanze, um die es sich bei Jona 4,6 handelt, wurde schon von Plinius des Älteren als ricinus identifiziert; S. AugustinusHieronymus, Epistular mutae I, Anm. 347, 229. 


\section{Wörtliche und sinngemäße Übersetzung}

Seine Überlegungen bezüglich dieser Angelegenheit schrieb Hieronymus schon in der frühen Phase seiner Tätigkeit als Übersetzer nieder. Das erste umfangreiche Werk, das er aus dem Griechischen ins Lateinische übertragen hatte, war das Buch der Chroniken des Eusebius von Cäsarea (um 380) ${ }^{69}$.

Wenn man die Sachlage berücksichtigt, dass es bisweilen zu den Pflichten des Übersetzers gehörte, den zu übersetzenden Text, unabhängig davon, um welche Literaturart es sich handelte, möglichst wortgetreu zu übertragen ${ }^{70}$, schienen die Überlegungen des Hieronymus eine Innovation zu sein. Es war ihm durchaus bewusst, dass die freie Übertragung, die jedoch den Sinn der Aussage nicht verändert, auf Widerstand stoßen und den Vorwurf einer Fälschung nach sich ziehen könnte.

Ähnlich wie im Fall der Übersetzung ad fontes sah sich unser Autor dazu gedrängt, diesbezüglich eine Rechenschaft gegenüber seinen Lesern abzulegen. So stoßen wir im Vorwort zu dem oben genannten Buch der Chroniken auf folgende Aussage:

[...] Es ist nämlich nicht einfach, bei wörtlicher Übertragung eines fremden Textes nicht mitunter doch davon abzuweichen, es kostet Mühe, das, was sich in der fremden Sprache gut anhört, mit der gleichen Eleganz in der Übersetzung festzuhalten. [...] Übersetze ich Wort für Wort, klingt es absurd; nehme ich, weil es nicht anders geht, an der Wortfolge oder Wortwahl irgendeine Änderung vor, wird es aussehen, als vernachlässigte ich meine Pflichten als Übersetzer ${ }^{71}$.

Aufgrund dieser Aussage lässt sich erkennen, dass Hieronymus die Übertragung ad sensum bevorzugt, obgleich sie eine gewisse Gefahr in sich birgt. Als Beispiel nennt er die ihm bekannten griechischen ATÜbersetzungen des Aquila, Symmachus und Theodotion, von denen die erste wortwörtlich, die zweite mehr sinngemäß und die dritte traditionsgetreu verfasst wurde. Für den unkundigen Leser, der nicht wisse, dass es sich um die Übertragungen aus dem Hebräischen handelt, wirkten sie au-

69 S. Fürst, Hieronymus, S. 80.

70 S.M. Mülke, Der Autor und sein Text. Die Verfälschung des Originals im Urteil antiker Autoren, Berlin 2008; A. Seele, Römische Übersetzer, Nöte, Freiheiten, Absichten. Verfahren des literarischen Übersetzens in der griechisch-römischen Antike, Darmstadt 1995.

71 Chronik, Vorwort, Übersetzung Fürst, Hieronymus, S. 259-262. 
fgrund der schmuddeligen sprachlichen Einkleidung ${ }^{72}$ eher abschreckend, sodass er sich keine Gedanken mehr über ihren Inhalt zu machen bemühe. Handelt es sich um die profane Literatur, so stellt Hieronymus dabei fest, dass die sprachliche Eleganz im Fall einer wörtlichen Übertragung verloren gehe $^{73}$. Dafür sprechen auch Beispiele, die Sophronius Eusebius im weiteren Textverlauf anführt, und sich dabei auf die Autorität Ciceros oder Horaz beruft ${ }^{74}$.

Bei weiterer Lektüre des Vorworts zu den Chroniken entdeckt man jedoch eine in diesem Kontext überraschende Äußerung:

[...] Man muss nämlich wissen, dass ich sowohl die Rolle des Übersetzers als auch in manchen Teilen die des Verfassers eingenommen habe. Denn ich habe einerseits den griechischen Text völlig getreu seinem Wortlaut wiedergegeben, andererseits manches, was mir zu fehlen schien, hinzugefügt ${ }^{75}$.

Eine gewisse Inkonsequenz zwischen Theorie und Praxis ist im Bezug auf das Thema Übersetzungsprinzipien des Öfteren in Werken des Hieronymus zu entdecken. Im Fall eines besonderen Briefes, der auch den Titel „De optimo genere interpretandi“ ${ }^{\text {“ }}$ trägt $^{16}$, kommt diese Diskrepanz insbesondere zum Vorschein. Es ist jedoch zu berücksichtigen, dass es sich dabei um eine Streitschrift handelt, die Hieronymus zu seiner Verteidigung verfasste, um die Vorwürfe einer vorsätzlichen Fälschung bei der Übertragung eines privaten Briefes aus dem Griechischen ins Lateinische, zurückzuweisen ${ }^{77}$.

Er gibt offen darin zu, bei der Übersetzung aus dem Griechischen dem Prinzip ad sensum nachzugehen, mit einer Ausnahme: „Ich gestehe und bekenne mit allem Freimut, dass ich bei der Übersetzung griechischer Texte, abgesehen von den Heiligen Schriften, wo selbst die Anordnung der Worte ein Geheimnis ist, nicht Wort für Wort, sondern sinngemäß übertrage" ${ }^{\text {"7 } 78}$.

Die Auffassung Ciceros, der bei seinen Übersetzungen nicht Wort für Wort übertrage, sondern den Geist und die Kraft der einzelnen Wörter beizubehalten versuche, damit sie auch seinem Sprachgefühl entspra-

72 Fürst, Hieronymus, S. 260.

73 Fürst, Hieronymus, S. 260.

74 Fürst, Hieronymus, S. 260.

75 Chronik, Vorwort, Übersetzung, Fürst, Hieronymus, S. 261.

76 Es handelt sich um Ep. 57 ad Pammachium aus dem Jahr 395/396.

77 S. Ep. 57, 1.2; Übersetzung L. Schade, BKV, https://www.unifr.ch/bkv/kapitel13348-1.htm.

78 Ep. 57, 5. Übersetzung Schade, BKV. 
chen $^{79}$ zitierend, scheint Hieronymus die Übersetzungs-prinzipien seines großen Vorbildes zu befürworten. Aber gelten sie genauso im Fall der Bibelübersetzung? Die eingefügte Einschränkung: ,abgesehen von den Heiligen Schriften“" würde darauf hinweisen, dass sich Hieronymus für die wörtliche Übersetzung der biblischen Texte ausspricht ${ }^{80}$. Bei der weiteren Lektüre des Briefes 57 überrascht der Autor jedoch mit Berufung auf das Vorwort zu seiner Chronik-Übersetzung, wo er sich ausdrücklich für die sinngemäße Übertragung aussprach ${ }^{81}$, um letzten Endes zu betonen, dass er: ,,[...]immer und schon von Jugend an gegen das Kleben am Worte war und nach dem Sinne übersetzte“"82.

Im darauf folgenden Kapitel führt Hieronymus zahlreiche Beispiele aus den Evangelien auf, die von dem griechischen Text der Septuaginta abweichen $^{83}$. Er weist auf die von den Evangelisten zugefügten Wörter und Sätze ${ }^{84}$ hin, auf die falsche Zitierung oder gar Verwechslung der Propheten ${ }^{85}$. Aufgrund der Tatsache, dass niemand die Evangelisten oder den Apostel Paulus wegen der freien, nicht wortgetreuen Übersetzungen einer Fälschung zu bezichtigen wagte, kommt unser Autor zu dieser Schlussfolgerung:

Aus allen diesen Beispielen ergibt sich einwandfrei, dass es den Aposteln und Evangelisten bei der Übersetzung der alten Schriften auf den Sinn ankam und nicht auf die Worte. Sie machten sich nicht viel Sorge um die Anordnung und um den Wortlaut, sofern nur das Verständnis nicht darunter litt ${ }^{86}$.

[...] Was meine Verleumder angeht, inzwischen mögen sie begreifen lernen, dass es in den Heiligen Schriften nicht auf die Worte, sondern auf den Sinn ankommt ${ }^{87}$.

79 Ep. 57, 5. Übersetzung Schade, BKV.

80 Eine Interpretation dieser rätselhaften Aussage bietet Weigert, Hebraica veritas, S. 48-52. S. auch G. Grützmacher, Hieronymus. Eine biographische Studie zur Alten Kirchengeschichte in 3 Bänden, Bd. 2, Aalen 1969, 109; Schade, Inspirationslehre, S. 138.

81 S. oben S. 11.

82 Ep. 57, 6.

83 Ep. 57, 7-10.

84 In diesem Fall handelt es sich um den Ausdruck aus Mk 5, 41 „Talitha qumi“, dem der Evangelist ,,ich sage dir“ beigefügt hatte, obwohl es dort bloß „Mädchen, steh auf!" steht. S. Ep. 57, 7.

85 Eins der vielen Beispiele bezieht sich auf die Stelle Mk 1,2-3, wo der zitierte Text nicht vom Jesaja stammt, wie Markus fälschlicherweise behauptet, sondern eine Zusammensetzung aus den Wörtern Maleachi und Jesaja darstellt. S. Ep. 57, 9.
86 Ep. 57, 9.
87 Ep. 57, 10. 
Soweit die Theorie und das Prinzip, das Hieronymus um das Jahr 396 vertrat. Was aber die Praxis angeht, so lesen wir z.B. in der Einleitung zum Buch Jona, das unser Autor um das Jahr 391/392 aus dem Hebräischen übersetzt hatte, dass er ,das Buch fast überall Wort für Wort übersetzt und dabei die Reihenfolge der Worte möglichst genau eingehalten hatte ${ }^{\text {“888 }}$.

Hieronymus selbst schreibt im Prolog zu seiner Hiob-Übersetzung (392/393), dass er bei seiner Arbeit keine früheren Vorlagen benutzt, sondern den Text teilweise wörtlich, teilweise sinngemäß, teilweise aber beides aus dem Hebräischen und Arabischen und an manchen Stellen auch aus dem Syrischen übertragen hätte ${ }^{89}$.

Zur gleichen Zeit etwa verfasste Sophronius Eusebius die Übersetzung des Buches Judit, die aufgrund von Zeitmangel, (sie soll während einer Nacht entstanden sein), „,mehr sinngemäß als Wort für Wort ${ }^{\text {‘990}}$ ausfiel. Von dem Buch Ester heißt es dagegen, dass es „eng Wort für Wort“ übertragen worden sei ${ }^{91}$.

Bei so vielen widersprüchlichen Aussagen scheint die Aufgabe, die richtige zu ermitteln erschwert zu sein. Im Fall der Übersetzungsprinzipien des Hieronymus beruft man sich deshalb auf die Praxis, die besagt, dass bei der Übertragung in eine fremde Sprache der Sinn der Aussage getroffen und weniger die Reihenfolge der Wörter beibehalten werden soll.

Bei der Verständnisfrage kommt der Übersetzer nicht um die Schwierigkeit der Ausdrucksweise herum. Bereits in einem Brief an den Papst Damasus ${ }^{92}$ aus dem Jahr 384 geht Hieronymus auf dieses Problem ein. Dort schildert er sein Bemühen bei der Übertragung der heiligen Schriften aus dem Hebräischen ins Lateinische die Einfachheit des Originals beizubehalten, um des Lesers Willen. Jegliche rhetorische Kunst oder poetische Verzierung wären dort entbehrlich, denn die Bibel mit ihrer Botschaft benötige einer einfachen, beinahe alltäglichen Sprache, die den Sinn erklären und die Dunkelheiten erhellen würde ${ }^{93}$. Die Übersetzung darf jedoch auf keinen Fall die Regel der Schönheit einer Sprache verletzen. Hieronymus selbst erlebte einst die Sprache der Propheten als abschreckend und ungepflegt $^{94}$, wenn man sie mit den antiken Autoren verglich. Daraufhin strebte Sophronius Eusebius im Laufe seiner translatorischen Beschäftigung nach

\footnotetext{
88 Comm. In poph. Ionam, Einleitung, FC 60, 27.

89 Prol. Ijob, Übersetzung Fürst, Hieronymus, S. 269-270.

90 Prol. Iudith, Übersetzung Fürst, Hieronymus, S. 89.

91 Prol. Hester, Übersetzung Fürst, Hieronymus, S. 89.

92 Es handelt sich um Ep. 36 entstanden um 384.

93 S. Ep. 36, 14.

94 S. Ep. 22, 30.
} 
der Vereinbarkeit von sancta simplicitas ${ }^{95}$ mit den Regeln des schönen, klassischen Lateins. Ausschlaggebend war dabei die Bewahrung des Sinnes einer Aussage: „Folgende Übersetzungsregel ist nämlich, wie wir schon oft gesagt haben, zu befolgen: Dass man dort, wo es keine Einschränkung im Sinn bedeutet, dem Wohlklang und dem eigenen Charakter der Sprache, in die wir übersetzen, treu bleibt" ${ }^{\text {"966. }}$.

Anhand der oben genannten Beispielen lässt sich eine Schlussfolgerung ziehen, dass obwohl Hieronymus ,,immer und schon von Jugend an gegen das Kleben am Worte war und nach dem Sinne übersetzte ${ }^{697}$, wenn die Lage es erforderte, durchaus im Stande war, eine wörtliche Übersetzung zu verfassen. Generell, wie auch im Fall der Übertragung aus der Quelle, galt, den Erwartungen der Leserschaft den Vorrang zu geben. Denn die wichtigste Aufgabe des Übersetzers und des Kommentators ist, laut Hieronymus, das, was dunkel und unverständlich scheint, klar und verständlich darzustellen ${ }^{98}$.

Der Eifer des Lesers soll dabei nicht abgeschreckt, sondern gefördert werden, sodass er nach und nach die Heiligen Schriften erforsche. Denn die Botschaft, die Hieronymus durch seine Arbeit vermitteln will, soll für jeden Christen erstrebenswert sein, denn: ,ignoratio scripturarum ignoratio Christi est“" ${ }^{\text {t69 }}$.

\title{
Zasada przekładu według Hieronima w teorii i praktyce
}

\author{
(streszczenie)
}

Hieronim ze Strydonu (347-419), jeden z czterech wielkich Ojców Kościoła Zachodniego przeszedł do historii jako thumacz Biblii. Jego zainteresowanie Pismem Świętym, oczytanie w dziełach wczesnych pisarzy chrześcijańskich oraz znajomość języka greckiego i hebrajskiego sprawiły, że był uważany przez jemu współczesnych za eksperta w dziedzinie egzegezy biblijnej. Dzięki korespondencji z papieżem Damazym zrodziła się idea rewizji wielu istniejących, różniących się od siebie przekładów Ewangelii na podstawie tekstów źródłowych. Wkrótce jednak rozpoznano konieczność rozszerzenia prac rewizyjnych również na księgi Starego Testamentu, które Hieronim postanowił tłumaczyć, opierając się na tekstach hebrajskich, a nie jak dotychczas na LXX. Innowacyjność tego przedsięwzięcia napotkała na krytykę i sprzeciw w wielu kręgach kościelnych. Obawiano się ogromnego wpływu, jaki Hieronim mógł wywrzeć na kształt Biblii dzięki swojemu nowemu przekładowi opierającemu się na oryginalnych tekstach źródłowych i zachowującemu sens przekazywanej zbawczej treści, a nie zachowującemu jej dosłownego brzmienia, co byłoby równoznaczne z detronizacją Septuaginty.
95 Ep. 57, 12.
96 Ep. 106, 55. Übersetzung Wiegert, Hebraica veritas, S. 64.
97 Ep. 57, 6.
98 Praef. in Ionam.
99 Comm. In Esaiam, Prol. 
Słowa kluczowe: Hieronim ze Strydonu; tłumacz Biblii; egzegeza biblijna; papież Damazy; Stary Testament

\title{
The Principle of Translation according to Jerome in Theory and Practice
}

\author{
(summary)
}

Jerome of Strydon (347-419), one of the four great Fathers of the Western Church, is remembered in history as a translator of the Bible. His interest in the Holy Scriptures, great knowledge of the works of early Christian writers and fluency in Greek and Hebrew meant that he was considered by the contemporaries as an expert in the field of biblical exegesis. Thanks to the correspondence with Pope Damas, the idea of revising many existing, differing translations of the Gospel based on source texts emarged. Soon, however, the necessity to expand the revision of the works was recognized, also to the books of the Old Testament, which Jerome decided to translate, based on the Hebrew texts, and not on the LXX as before. The innovation of this undertaking encountered criticism and opposition in many church circles. There was a fear of the enormous influence that Jerome might have had on the shape of the Bible thanks to his new translation based on original source texts and preserving the meaning of salvation content rather than preserving its literal sound, which would be the dethronement of the Septuagint.

Keywords: Jerome of Strydon; translator of the Bible; biblical exegesis; Pope Damas; Old Testament

\section{Bibliographie}

\section{Bibeltexte}

Biblia Sacra Vulgata, red. R. Weber - R. Gryson, Stuttgart 2007.

\section{Quellen}

Augustinus - Hieronymus, Epistulae mutae, lateinisch-deutsch = Briefwechsel/ Augustinus - Hieronymus, übers. A. Fürst, FC 41, Turnhout 2002.

Hieronymus, Chronicon, red. R. Helm, GCS 47, Eusebius Werke VII, Berlin 1984.

Hieronymus, Commentarius in Ionam prophetam, lateinisch-deutsch $=$ Kommentar $\mathrm{zu}$ dem Propheten Jona / Hieronymus, übers. S. Risse, FC 60, Turnhout 2003.

Hieronymus, Epistula 57 ad Pammachium, BKV, red. L. Schade, https://www.unifr.ch/ bkv/kapitel3348.htm

Hieronim, List 57 do Pammachiusza o najlepszym sposobie tlumaczenia, tł. J. Czuj, ZMT, Krakow 2010, 57-68. 
Hieronymus, Opera exegetica 2, Commentariorum in Esaiam Libri I-XI, red. M. Adriaen, CChr. SL 73, Turnhout 1963.

\section{Sekundärliteratur}

Birnbaum E., Der Koheletkommentar des Hieronymus, Einleitung, revidierter Text, Übersetzung und Kommentierung, CSEL Extra Seriem, Berlin - Boston 2014.

Durst M., Art. Hieronymus, LThK 5, 91-93.

Brown D., Vir Trilinguis. A Study in the Biblical Exegesis of Saint Jerome, Kampen 1992.

Fürst A., Kürbis oder Efeu?, BN 72 (1994) 12-19.

Fürst A., Art. Hieronymus, LACL, Freiburg in Breisgau 2002, 323-331.

Fürst A., Hieronymus. Askese und Wissenschaft in der Spätantike, Freiburg im Breisgau 2003.

Grützmacher G., Hieronymus - Eine biographische Studie zur alten Kirchengeschichte in 3 Bänden, SGTK 10, Aalen 1969.

Hennings R., Der Briefwechsel zwischen Augustinus und Hieronymus und ihr Streit um die Auslegung von Gal. 2, 11-14, VCSup 21, Leiden 1994.

Kramer B., Art. Didymos der Blinde, LThK 3, 213.

Mülke M., Der Autor und sein Text. Die Verfälschung des Originals im Urteil antiker Autoren, Berlin 2008.

Opelt I., Hieronymus `Streitschriften, Heidelberg 1973.

Rebenich S., ,, Vir Trilinguis“ and the „Hebraica Veritas”, VigChr 47 (1993) 50-77.

Reventlow Graf H., Epochen der Bibelauslegung, Bd. II. Von der Spätantike bis zum angehenden Mittelalter, München 1994.

Schade L., Die Inspirationslehre des Heiligen Hieronymus. Eine biblisch-geschichtliche Studie, BSt 15, Freiburg 1910.

Schulz-Flügel E., Hieronymus, Feind und Überwinder der Septuaginta? Untersuchungen anhand der Arbeiten an den Psalmen, in: Septuaginta-Psalter und seine Tochterübersetzungen, red. A. Aejmelaeus - U. Quast, MSU 24, Göttingen 2000, 33-50.

Schulz-Flügel E., Hieronymus - Gottes Wort. Septuaginta oder hebraica Veritas, in: red. W. Kraus - S. Kreuzer, Die Septuaginta - Text, Wirkung, Rezeption, WUNT 325, Tübingen 2004, 746-758.

Weigert S., Hebraica Veritas. Übersetzungsprinzipien und Quellen der Deuteronomiumübersetzung des Hieronymus, BWANT 11/7, Stuttgart 2016. 